\title{
Predictive Value of MRI in Diagnosing Brain AVM Recurrence after Angiographically Documented Exclusion in Children
}

\author{
(D) A. Jhaveri, (D) A. Amirabadi, (D) P. Dirks, (D)A.V. Kulkarni, (D) M.M. Shroff, (D) N. Shkumat, (D). Krings, (D).M. Pereira, (D) V. Rea, and \\ (iD). Muthusami
}

\begin{abstract}
BACKGROUND AND PURPOSE: MRI is routinely performed following brain AVM after treatment in children. Our aim was to determine the predictive values of contrast-enhanced MR imaging and TOF-MRA for brain AVM recurrence in children, compared with conventional angiography and the role of 3D rotational angiography-MR imaging fusion in these cases.
\end{abstract}

MATERIALS AND METHODS: We included all pediatric patients with brain AVMs during an 18-year period with angiographically documented obliteration after treatment. Patients underwent 3T MR imaging, including contrast-enhanced MR imaging, TOF-MRA, and conventional angiography, with a subset undergoing 3D rotational angiography. The predictive values of contrast-enhanced MR imaging and TOF-MRA for brain AVM recurrence were determined. CTA sections reconstructed from 3D rotational angiography were coregistered with and fused to 3D-TIWI for analysis.

RESULTS: Thirty-nine children (10.8 \pm 3.9 years of age; range, 2-17 years; male/female ratio, 19:20; mean Spetzler-Martin grade, 1.9 \pm 0.6 ) met the inclusion criteria. Of these, 13 had angiographically confirmed brain AVM recurrence, 8 following surgery and 5 following embolization. Sensitivity, specificity, and positive and negative predictive values for recurrence were the following: contrast-enhanced MR imaging: 84.6\%, 38.5\%, 40.7\%, 81.8\%; TOF-MRA: 50.0\%, 96.1\%, 85.7\%, 79.3\%; both: 75.0\%, 90.9\%, 85.7\%, 83.3\%. 3D rotational angiography-MR imaging fused images confirmed or excluded recurrence in all available cases (13/13). Embolization-only treatment was a significant predictor of recurrence $(O R=32.4, P=.006)$. MR imaging features predictive of recurrence included a tuft of vessels on TOF-MRA and nodular juxtamural/linear enhancement with a draining vein on contrast-enhanced MR imaging.

CONCLUSIONS: MR imaging is useful for surveillance after brain AVM treatment in children, but conventional angiography is required for definitive diagnosis of recurrence. TOF-MRA and contrast-enhanced MR imaging provide complementary information for determining brain AVM recurrence and should be interpreted in conjunction. 3D rotational angiography-MR imaging fusion increases the diagnostic confidence regarding brain AVM recurrence and is therefore suited for intraoperative neuronavigation.

ABBREVIATIONS: $\mathrm{bAVM}=$ brain AVM; $C A=$ conventional angiography; CEMRI = contrast-enhanced MR imaging; $3 \mathrm{DRA}=3 \mathrm{D}$ rotational angiography; $\mathrm{NPV}=$ negative predictive value; $P P V=$ positive predictive value; $S M=$ Spetzler-Martin

$\mathbf{T}$ he annual risk of brain arteriovenous malformation (bAVM) rupture is estimated at $2 \%-4 \%$, with a $25 \%$ mortality rate per event. ${ }^{1-3}$ In children, bAVMs could remain undetected but usually present with rupture, accounting for most childhood intra-

Received April 2, 2019; accepted April 18.

From Pediatric Neuroradiology and Image Guided Therapy (A.J., A.A., M.M.S., N.S., V.R., P.M.), Diagnostic Imaging, and Division of Neurosurgery (P.D., A.V.K.), Department of Surgery, The Hospital for Sick Children, University of Toronto, Toronto, Ontario, Canada; and Divisions of Neuroradiology and Neurosurgery (T.K.,V.M.P.), Departments of Medical Imaging and Surgery, University of Toronto, and Toronto Western Hospital, University Health Network, Toronto, Ontario, Canada.

Please address correspondence to Prakash Muthusami, MD, Diagnostic Imaging, IGT, The Hospital for Sick Children, 555 University Ave, Toronto, ON - M5G 1X8, Canada; e-mail: Prakash.muthusami@sickkids.ca; @PMuthusami

三 Indicates article with supplemental on-line table.

http://dx.doi.org/10.3174/ajnr.A6093 cranial hemorrhages. ${ }^{4,5}$ Presentation with hemorrhage is a wellknown risk factor for future bleeding from a bAVM. ${ }^{1,6,7}$ Modern treatment of bAVM comprises multimodality interventions alone or in combination: operation, stereotactic radiosurgery, and embolization. The results of A Randomized Trial of Unruptured Brain AVMs (ARUBA), ${ }^{8}$ which favored conservative management over active intervention for unruptured brain AVMs in adult patients, have been the subject of much debate. ${ }^{9-13}$ However, for ruptured bAVMs, complete angiographic obliteration is the accepted standard of care, due to a known increased risk of a second hemorrhage.

Recurrence after treatment of bAVMs in children is reported in $5 \%-15 \%$ of cases, which is higher than in adult patients. ${ }^{4,14}$ This necessitates angiographic documentation of complete obliteration, as well as close follow-up. There is no consensus regard- 
ing the optimal timing and imaging technique for surveillance, with MR imaging, CTA, and conventional angiography (CA) playing an important role in the literature. ${ }^{5,15,16} \mathrm{CA}$ is the criterion standard to document shunt obliteration, visualize recurrence, and tailor appropriate treatment in bAVMs. However, given its invasive nature, the potential risk of complications, need for general anesthesia in children, and exposure to ionizing radiation, there is interest in developing noninvasive methods that can yield similar results. Pediatric patients at our institution are followed up with MR imaging, with CA performed in case of abnormal MR imaging findings, and at 1 year in case of normal MR imaging findings. In cases with small recurrences or slow shunting, 3D rotational angiography is performed at the discretion of the interventional neuroradiologist, with subsequent fusion to 3D MR imaging (3DRA-MRI).

With the sensitivity of MRA being low for small recurrences, ${ }^{17-20}$ contrast-enhanced MR imaging (CEMRI) is also performed as part of routine follow-up. However, CEMRI performed postsurgically is subject to false-positive enhancement related to reactive postoperative tissue along surgical margins or enhancement of dural folds within a hemorrhagic cavity or surgical bed. ${ }^{21,22}$ Additionally, there is emerging evidence in recent years of gadolinium deposition, ${ }^{23,24}$ with a concern for brain toxicity with repeat administrations. ${ }^{25}$ It is therefore imperative that gadolinium be administered only when there is definite benefit, ${ }^{26}$ especially for diseases like bAVM that require long-term follow-up. The adequacy of MR imaging for predicting recurrence of completely treated brain AVMs in children has not been studied, to the best of our knowledge. The additional benefit of performing CEMRI has also not been evaluated.

The primary objective of this study was to determine the predictive value of MR imaging as a surveillance tool for bAVM recurrence after angiographically documented obliteration in children. Secondary objectives were to assess whether CEMRI provided additional information not obtained from noncontrast MRA in this population and to assess whether 3D rotational angiography with MR imaging fusion increased diagnostic confidence for bAVM recurrence in children.

\section{MATERIALS AND METHODS Study Design}

An institutional review board-approved retrospective study was undertaken in 236 consecutive patients with bAVMs, 18 years of age and younger, managed at the Hospital for Sick Children, Toronto, from January 2000 to February 2018. Currently, we follow a protocol of performing MR imaging with TOF-MRA and CEMRI at 3 months, 6 months, and 1 year following angiographically documented cure, with a conventional angiography performed at approximately 1 year or at any subsequent time point when there is suspicion of recurrence on surveillance MR imaging. Following a posttreatment 1 -year CA with negative findings, children are followed up at our institution with MR imaging at increasing intervals until 18 years of age, after which time they are transitioned to an adult neurovascular service for continued follow-up. For this study, patients with reported complete cure following any therapeutic method including embolization, surgical excision, radiosurgery, or multimodal therapy were included, with the first negative posttreatment angiography findings as a baseline. In addition, for historical cases treated before our current protocol, to be included in the study, patients had to have a posttreatment follow-up 3T MR imaging examination, which included a TOF-MRA and/or CEMRI, as well as a final CA examination at approximately 1 year following treatment, with these 2 examinations being no more than 6 months apart. Patients were excluded if they were managed conservatively $(n=34)$, had no intraoperative/posttreatment angiography $(n=18)$, no posttreatment follow-up 3T MR imaging available $(n=101)$, no documented cure after gamma knife treatment $(n=26)$ or follow-up CA was performed $>6$ months after follow-up MR imaging $(n=18)$.

For included cases $(n=39)$, data were collected on the following parameters: clinical details (patient sex and age, primary presentation), bAVM features (location, feeding arteries, and type: terminal or en passant or both; venous drainage: superficial or deep or both; presence of intranidal or postnidal aneurysms; presence of venous stenosis, Spetzler-Martin grade, and a compact/ diffuse nidus), treatment details (treatment type: operation, endovascular, gamma knife, or combinations of these) and date, interval between treatment and first follow-up MR imaging, recurrence on TOF-MRA and CEMRI, and finally, the post-MR imaging CA interval and the presence of recurrence on CA and on 3DRA-MR imaging if performed. The presence of bAVM recurrence on CEMRI, TOF-MRA, and 3DRA-MR images, as well as descriptors for recurrence, were determined by the study neuroradiologist (P.M.), with analysis of PACS reports by another investigator (A.J.), blinded to each other's findings. Cases with discrepant findings were re-evaluated by another neuroradiologist (M.M.S.) to reach a consensus.

\section{Imaging}

MR imaging was performed on a 3T magnet (Magnetom Skyra, Siemens, Erlangen, Germany; or Achieva, Philips Healthcare, Best, the Netherlands) with a 32-channel transmit-receive head coil. The examination included standard T1-, T2-, and FLAIRweighted sequences, followed by TOF-MRA with the following parameters: $\mathrm{TR}=25 \mathrm{~ms}, \mathrm{TE}=3.81 \mathrm{~ms}$, flip angle $=18^{\circ}$, bandwidth $=186 \mathrm{~Hz}$, matrix size $=384 \times 274$, FOV $=180 \times 180$ $\mathrm{mm}^{2}$, resulting in an in-plane spatial resolution of $0.2 \times 0.2 \mathrm{~mm}^{2}$ with 0.5 -mm slice thickness. 3D-T1-turbo field echo images were acquired before and after the administration of $0.1 \mathrm{mmol} / \mathrm{kg}$ of gadolinium-based contrast agent (gadobutrol, Gadovist; Bayer Schering Pharma, Berlin, Germany) with the following parameters: $\mathrm{TR}=2200 \mathrm{~ms}, \mathrm{TE}=3 \mathrm{~ms}, \mathrm{TI}=900 \mathrm{~ms}$, flip angle $=9^{\circ}$, bandwidth $=240 \mathrm{~Hz}$, matrix size $=224 \times 224$, FOV $=220 \times 220$ $\mathrm{mm}^{2}$, resulting in an in-plane spatial resolution of $1.0 \times 1.0 \mathrm{~mm}^{2}$ with a $0.98-\mathrm{mm}$ slice thickness. Conventional angiography was performed by 1 of 2 interventional neuroradiologists (P.M. or M.M.S.), either in a biplane neuroangiography suite (Artis Q BA Twin; Siemens) or intraoperatively in a hybrid suite (Artis Zeego; Siemens).

All procedures were performed with the patient under general anesthesia through femoral artery access. Angiography was performed using a $4 \mathrm{~F}$ diagnostic catheter with standard projections, followed by rotational angiography being performed in the arte- 
rial distribution of interest at the discretion of the operator, using the following parameters: 5-second rotational acquisition generating 133-248 projections for a total coverage of $200^{\circ}$, with a detector dose/frame of $100-360 \mu \mathrm{Gy}$, reconstructions with a 512matrix algorithm $\left(0.20-\mathrm{mm}^{3}\right.$ voxel size), iodinated contrast agent (iohexol, Omnipaque $300 \mathrm{mg} \mathrm{I} / \mathrm{mL}$; GE Healthcare, Piscataway, New Jersey) injected through a power injector at $3-5 \mathrm{~mL} / \mathrm{s}$ depending on the age and arterial size, 1 - to 2 -second $\mathrm{x}$-ray acquisition delay after the start of contrast injection. For subtracted 3DRA, full-strength contrast was injected, whereas for unsubtracted 3DRA, contrast was diluted to $20 \%$ to reduce the total contrast volume and streak artifacts. Multiplanar and volumerendered reformats were performed on a dedicated workstation (syngo MultiModality Workplace; Siemens) by a dedicated technologist (V.R.), supervised by the operating neuroradiologist. A $3 \mathrm{D}$ set of CT angiographic images was reconstructed from these images, which were subsequently fused to the patient's pregadolinium 3D T1 MR image set, yielding 3DRA-MR images.

\section{Statistical Analysis}

Statistical analysis was performed using the SPSS statistical software, Version 24 (IBM, Armonk, New York). Categoric variables were expressed as numbers and percentages. Continuous variables were described using median and interquartile range or mean and SD as appropriate. The $\chi^{2}$ or Fisher exact test was used for evaluation of the association between the categoric variables, as appropriate. The sensitivity, specificity, and positive and negative predictive values of TOF-MRA and CEMRI were calculated using conventional angiography as the criterion standard. The diagnostic confidence scores for recurrence on MR imaging with 3DRA-MR imaging were compared using the Wilcoxon signedrank test. The generalized score statistic test, based on generalized estimation equations, was used to compare the predictive values of MR imaging and DSA. Univariate and multiple logistic regression analyses were performed to evaluate the AVM risk factors. A 2-tailed $P<.05$ was considered statistically significant.

\section{RESULTS}

\section{Patient Characteristics and Presentation}

Thirty-nine children with bAVMs fulfilled the inclusion criteria for the study. The mean age of our patient cohort was $10.8 \pm 3.9$ years (range, 2-17 years). Of these, 20 (51.3\%) were female, and 3 $(7.7 \%)$ had a diagnosis of hereditary hemorrhagic telangiectasia. Hemorrhagic presentation was seen in 28/39 (71.2\%) patients. Most $(24 / 39,61.5 \%)$ bAVMs in our patient cohort were SpetzlerMartin (SM) grade II (mean SM grade $=1.9 \pm 0.6$; range, I-IV). Terminal and en passant feeders were seen with similar frequency. Most bAVMs $(31 / 39,79.5 \%)$ had superficial venous drainage only, with venous stenosis seen in a minority $(3 / 39,7.7 \%)$. Clinical details and bAVM characteristics are summarized in Table 1.

\section{Treatment}

Treatment modalities received are shown in Table 1. All patients with bAVMs at our institution are discussed following initial diagnostic conventional angiography for management at a combined neurovascular conference with vascular neurosurgeons and
Table 1: Patient demographics in our cohort of pediatric bAVMs $(N=39)^{\mathrm{a}}$

\begin{tabular}{|c|c|}
\hline Demographics & \\
\hline Age (mean) (range) (yr) & $10.8 \pm 3.9,2-17$ \\
\hline Male/female ratio & $19 / 20$ \\
\hline \multicolumn{2}{|l|}{ Presentation } \\
\hline Hemorrhage & $28(71.2)$ \\
\hline Seizures & $5(12.8)$ \\
\hline HHT screening & $3(7.7)$ \\
\hline Incidental (screening for headache) & $2(5.1)$ \\
\hline Partially treated at outside institution & $1(2.6)$ \\
\hline \multicolumn{2}{|l|}{ SM grade } \\
\hline 1 & $10(26)$ \\
\hline II & $24(61.5)$ \\
\hline III & $4(10.3)$ \\
\hline IV & $1(2.6)$ \\
\hline \multicolumn{2}{|l|}{ Location } \\
\hline Frontal & $5(12.8)$ \\
\hline Parietal & $9(23.1)$ \\
\hline Temporal & $11(28.2)$ \\
\hline Occipital & $9(23.1)$ \\
\hline Brain stem & $1(2.6)$ \\
\hline Cerebellar & $3(7.7)$ \\
\hline Choroidal & $1(2.6)$ \\
\hline \multicolumn{2}{|l|}{ Arterial feeder type } \\
\hline Terminal & $19(48.7)$ \\
\hline En passant & $20(51.3)$ \\
\hline \multicolumn{2}{|l|}{ Venous drainage } \\
\hline Superficial only & $31(79.5)$ \\
\hline Deep ( \pm superficial drainage) & $8(20.5)$ \\
\hline \multicolumn{2}{|l|}{ Venous stenosis } \\
\hline Present & $3(7.7)$ \\
\hline Absent & $36(92.3)$ \\
\hline \multicolumn{2}{|l|}{ Compactness } \\
\hline Compact & $30(76.9)$ \\
\hline Diffuse & $9(23.1)$ \\
\hline \multicolumn{2}{|l|}{ Treatment } \\
\hline Surgery & $20(51.3)$ \\
\hline Embolization & $11(28.2)$ \\
\hline Radiosurgery & $3(7.7)^{\prime}$ \\
\hline Multimodality & $5(12.8)$ \\
\hline
\end{tabular}

Note:- HHT indicates hereditary hemorrhagic telengiectasia.

${ }^{a}$ Data are numbers (\%) unless otherwise indicated.

interventional neuroradiologists, and subsequently with the family. Most (20/39, 51.3\%) bAVMs in our cohort, being small and compact, were amenable to surgical resection. Endovascular treatment was performed in the following manner: 1) as standalone treatment in $11 / 39$ (28.2\%), when associated with a terminal feeder; 2 ) presurgically in $2 / 39(5.1 \%)$ to exclude a nonresectable nidus; 3 ) presurgically in $2 / 39(5.1 \%)$ to target the rupture point in acute hemorrhage; or 4) to reduce nidal volume before radiosurgery in $1 / 39(2.6 \%)$. Liquid embolics were used for all endovascular treatments, $n$-BCA (Histoacryl; Braun, Melsungen, Germany) with Lipiodol (Guerbet, Roissy, France) in 8/11 (72.7\%); and Onyx (Covidien, Irvine, California) in $3 / 11$ $(27.3 \%)$. One patient received additional coil occlusion of a fistulous feeder. Only 3 patients with completed radiosurgical treatment met the inclusion criteria for this study.

\section{Recurrent bAVM, Retreatment, and Recurrent Hemorrhage}

Recurrence was confirmed by CA in 13 patients, following treatment with embolization in 8 patients, and by surgical ex- 
cision in 5. No significant difference was noted between this group and those without recurrence with respect to age (10.4 \pm 3.4 versus $11.0 \pm 4.2$ years, $P=.67)$ or $\mathrm{SM}$ grade $(1.7 \pm 0.6$ versus $2.0 \pm 0.6, P=.20)$. The mean interval between treatment and confirmation of recurrence was $43.8 \pm 41.1$ months (range, 9.1-156.9 months), with a trend toward longer recurrence-free intervals after surgical-versus-endovascular treatment $(69.7 \pm 60.5$ versus $27.6 \pm 16.8$ months, $P=.08)$. Four of these recurrences were diagnosed by MR imaging within 6 months of treatment, 2 of which were true-positives on con-

Table 2: Results of multivariable logistic regression model to predict bAVM recurrence

\begin{tabular}{lcc}
\hline & $\begin{array}{c}\text { Odds Ratio } \\
(95 \% \mathrm{Cl})\end{array}$ & $\begin{array}{c}\boldsymbol{P} \\
\text { Value }\end{array}$ \\
\hline Age at diagnosis (yr) & $0.9(0.7-1.3)$ & .914 \\
Sex (M) & $3.3(0.4-28.7)$ & .287 \\
SM grade & $0.2(0.0-1.2)$ & .079 \\
Diffuse nidus (compact vs diffuse) & $0.2(0.0-2.8)$ & .256 \\
Draining vein stenosis (yes vs no) & $1.6(0.0-130.3)$ & .831 \\
Embolization only treatment & $32.4(2.7-386.3)$ & .006 \\
$\quad$ (yes vs no) & & \\
\hline
\end{tabular}

ventional angiography and underwent subsequent complete surgical resection. There were no rebleeds in our cohort. Multivariable analysis (Table 2) for predictors of bAVM recurrence showed no significant association with age, sex, SM grade, compactness of the nidus, type of feeder (terminal/en passant), presence of deep venous drainage, or presence of venous stenosis. However, we found a significant association of recurrence with embolization-only treatment $(\mathrm{OR}=32.4 ; 95 \% \mathrm{CI}$, 2.7-386.3; $P=.006$ ), which was in line with the results of univariate analysis $(\mathrm{OR}=12.27 ; 95 \% \mathrm{CI}, 2.4-63.4 ; P=.003)$. This was independent of the type of embolic agent used. The multivariable model explained $47.0 \%$ (Nagelkerke $R^{2}$ ) of the variance in outcome and correctly classified $79.5 \%$ of cases. Because the type of feeder (terminal versus en passant) was significantly associated with the SM grade (Fisher Exact test, $P=.006)$ and with the type of nidus (compact versus diffuse) (Fisher Exact test, $P=.02$ ), it was eliminated from our regression model.

Recurrence was diagnosed by CEMRI as enhancement with a sensitivity of $11 / 13(84.6 \%)$ and specificity of $10 / 26(38.5 \%)$, and
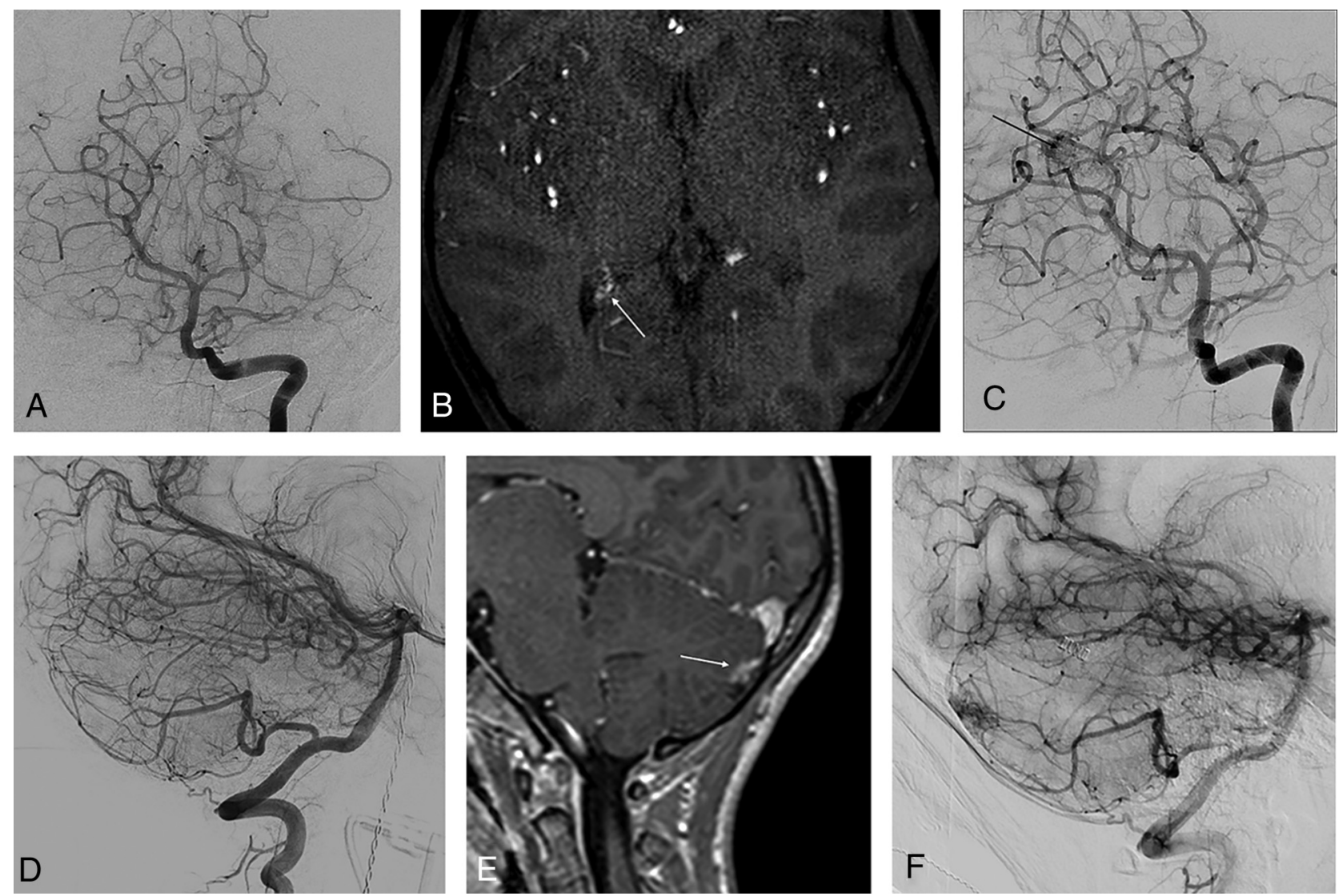

FIG 1. MR imaging appearances of true-positive AVM recurrence. A, Recurrent nidus in a 7-year-old girl with a right choroidal AVM that was embolized with $n$-BCA through a posterolateral choroidal feeder, with postembolization digital subtraction angiography confirming exclusion. $B$, Axial section from TOF-MRA performed 6 months posttreatment showing a tuft of vessels (arrow) posterior to the right lateral ventricular choroid plexus. This was also seen on contrast-enhanced MR imaging (not shown here) as exuberant and asymmetric choroid enhancement. $C$, Conventional angiogram, obtained 3 weeks after MR imaging, confirms an AVM nidus. Left vertebral artery injection confirms a right choroidal nidus (arrow) with deep venous drainage. The patient subsequently underwent radiosurgery. D, Recurrent cerebellar AVM in a 11-year-old girl with hereditary hemorrhagic telengiectasia lateral angiogram with negative findings immediately following endovascular embolization through a cerebellar branch of the right posterior inferior cerebellar artery. E, Sagittal section from postgadolinium MR imaging 3 months after treatment shows a nodular juxtamural focus of enhancement (arrow), with a vein traceable to the torcular. $F$, Conventional angiogram performed 1 month after MR imaging confirms this to be a recurrent nidus with early venous drainage. The recurrent AVM was surgically removed. 
on TOF-MRA, as abnormal vessels with a sensitivity of $6 / 12(50 \%$, performed after contrast in 1 case) and specificity of 25/26 (96.1\%). Positive and negative predictive values (PPV, NPV) for bAVM recurrence were CEMRI, $40.7 \%$ and $81.8 \%$, and TOFMRA, $85.7 \%$ and $79.3 \%$. Looking specifically at the 13 recurrences (On-line Table), we found that MR imaging features predictive of recurrence included a tuft of vessels on TOF-MRA (Fig $1 A-C)$, nodular juxtamural enhancement, or linear enhancement associated with a traceable draining vein on CEMRI (Fig 1D-F). When concordance of CEMRI and TOF-MRA findings was considered to make or exclude a recurrence, we found a sensitivity of $75 \%$, specificity of $90.9 \%$, PPV of $85.7 \%$, and NPV of $83.3 \%$

Table 3: Sensitivity, specificity, PPV, and NPV for CEMRI, TOFMRA, and a combination of both to diagnose recurrent brain AVM after treatment in children

\begin{tabular}{lccc}
\hline & CEMRI & TOF-MRA & CEMRI + TOF-MRA \\
\hline Sensitivity & $84.6 \%$ & $50.0 \%$ & $75.0 \%$ \\
Specificity & $38.5 \%$ & $96.1 \%$ & $90.9 \%$ \\
PPV & $40.7 \%$ & $85.7 \%$ & $85.7 \%$ \\
NPV & $81.8 \%$ & $79.3 \%$ & $83.3 \%$ \\
\hline
\end{tabular}

(Table 3). Of the 13 recurrences, 8 underwent subsequent surgical resection; 4 were referred for radiosurgery; and 1 , for endovascular embolization.

\section{DRA-MR Imaging Fusion for Confirmation of bAVM \\ Recurrence}

3DRA was performed in 14/39 (35.9\%) patients, being technically adequate in 13/14 (92.9\%) patients (suboptimal bolus timing in 1 patient). This yielded 13 patients for fusion with nongadolinium 3D T1 MR images. Although there were no additional findings or missed diagnoses on 2D-DSA, 3DRA-MR imaging information showed conclusively the presence of a nidus and abnormal/dysplastic vessels with contrast in a draining vein in 4 cases, of which 2 had been false-negative for recurrence on prior MR imaging (Fig 2 ), and conclusively excluded the presence of abnormal vascularity in an encephalomalacic cavity in 10 cases, of which 9 had shown false-positive enhancement on prior MR imaging (Fig 3). These "fused" 3D images, clearly defining the location and margins of the recurrent nidus, feeders, and draining veins, were used
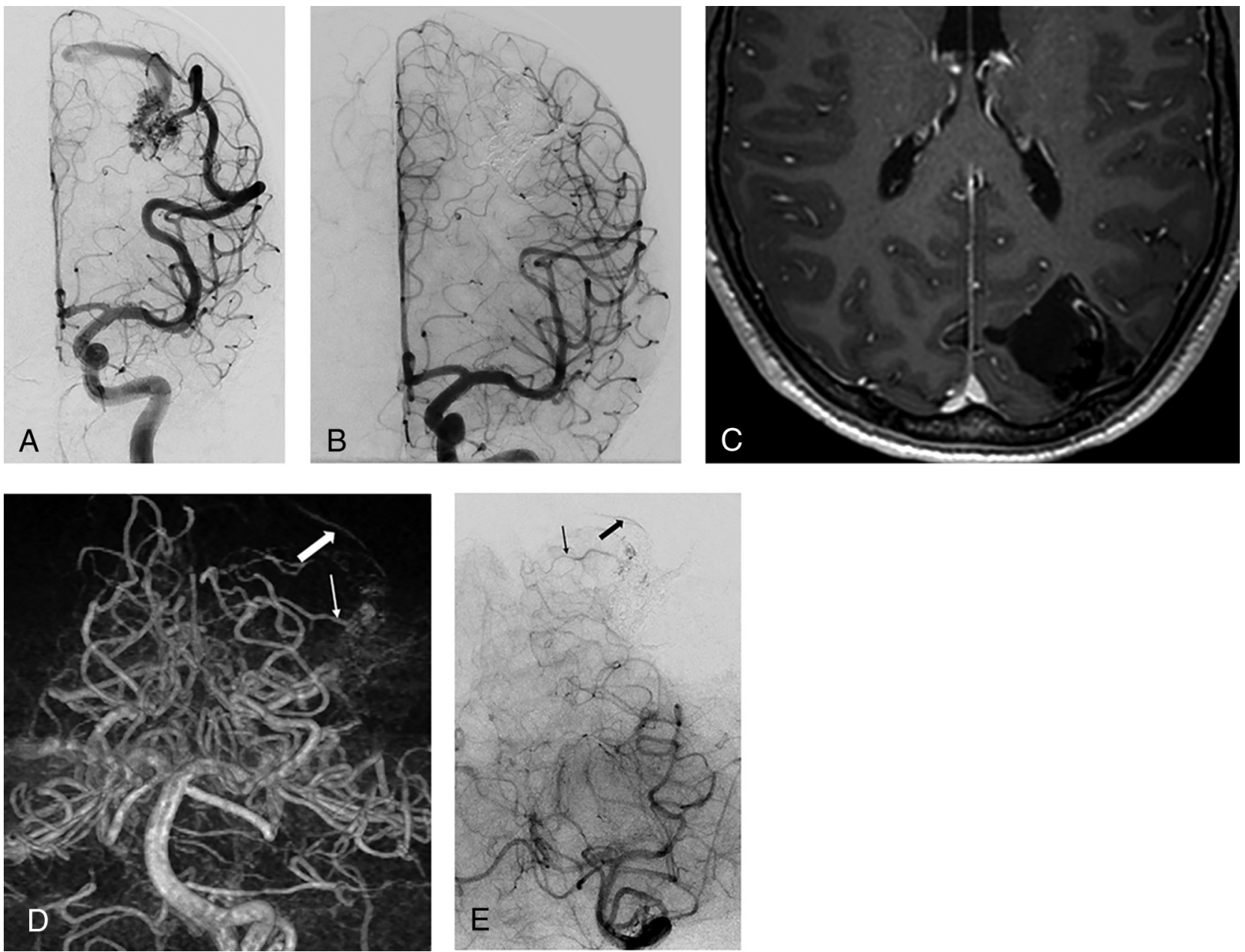

FIG 2. False-negative MR imaging findings for brain AVM recurrence, confirmed by digital subtraction and 3D rotational angiography. A 16-year-old boy with a left occipital AVM treated by embolization with Onyx. A, Frontal projection, left internal carotid angiography, shows a hypertrophied temporo-occipital middle cerebral artery branch suppling a compact nidus, with superficial venous drainage. $B$, Postembolization frontal angiogram shows complete exclusion of the shunt. $C$, Axial postgadolinium MR imaging section obtained 1 year after angiographically documented cure shows the hematoma cavity with artifacts from the Onyx cast within, but no suggestion of recurrence. $D$, Volume-rendered reformat obtained from 3D rotational angiography in the left vertebral artery shows a nidus behind the Onyx cast, with a prominent feeder (thin arrow) and early draining vein (thick arrow), which was also confirmed on digital subtraction angiography (E). 

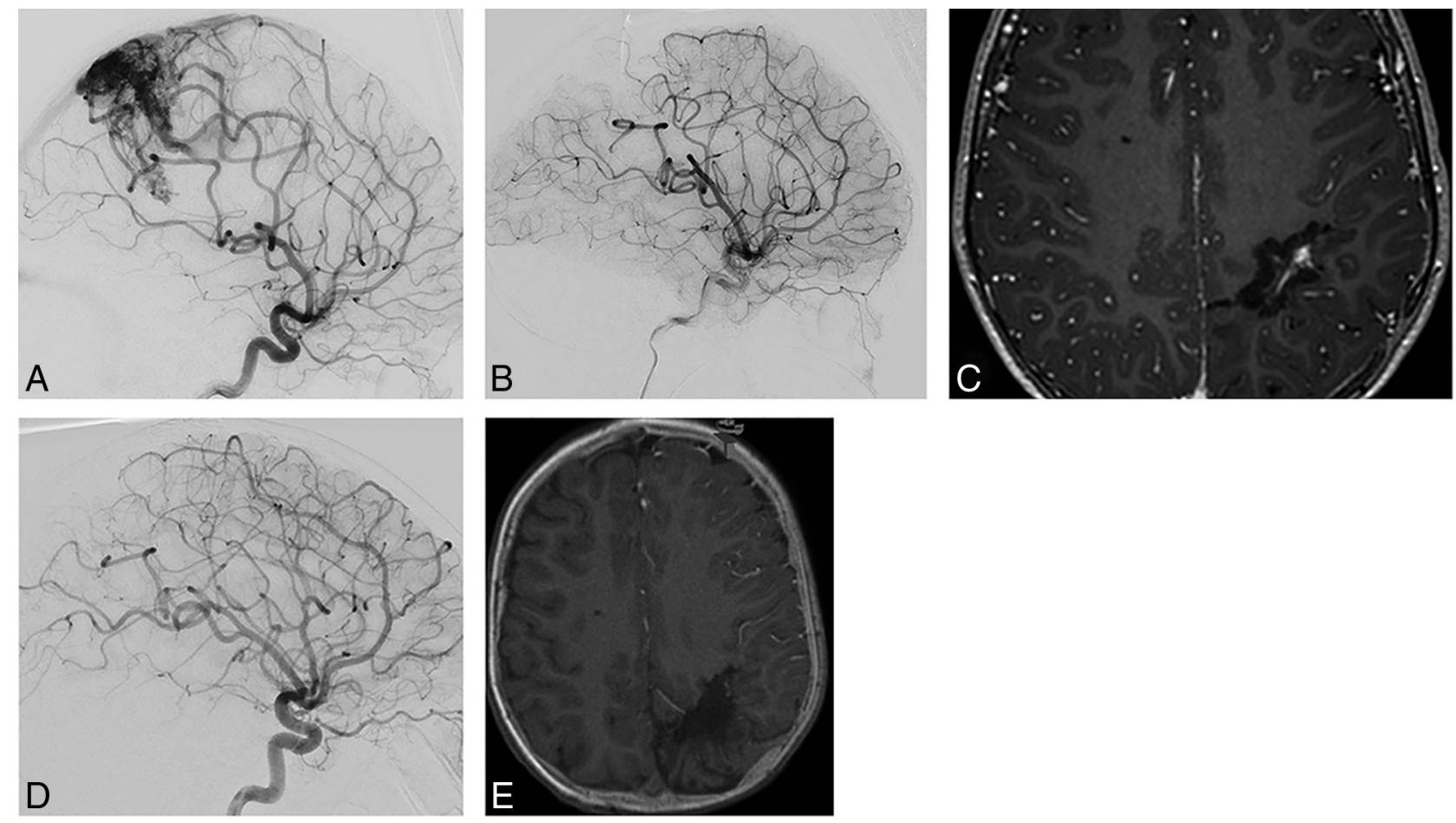

FIG 3. False-positive MR imaging for brain AVM recurrence, confirmed by conventional angiography and 3DRA-MR imaging fusion. A 5-year-old boy with a left parietal AVM. A, Left internal carotid injection in the lateral projection during conventional angiography shows a postcentral diffuse AVM nidus with a deep white matter component, hypertrophied anterior and middle cerebral arterial feeders, and venous drainage into the superior sagittal sinus. $B$, Complete surgical resection was performed after partial embolization, with cure confirmed with postresection angiography. C, Axial postgadolinium section from MR imaging performed 1 year after cure shows central linear and nodular enhancement suspicious for recurrence. $D$, Digital subtraction angiographic image, left internal carotid injection, shows no AVM recurrence. This was also confirmed from injections into the posterior circulation. E, 3DRA-MR imaging fusion shows no vascularity within the encephalomalacic cavity, excluding recurrence. MR imaging enhancement is believed to be related to scar tissue or dural folds.

for neuronavigation in 2 of these 4 recurrent cases that subsequently were resected (Fig 4).

\section{DISCUSSION}

Complete excision of the bAVM nidus with obliteration of arteriovenous shunting is required to eliminate the risk of hemorrhage. Although there is little in the neurosurgical literature about recurrent bAVMs, with sporadic case reports and series describing bAVM recurrence after angiographic confirmation of obliteration, ${ }^{27-29}$ they are likely more common than previously thought. A recent meta-analysis of this literature showed that bAVM recurrence occurred in $2.7 \%$ of reported adult series and in nearly $10 \%-15 \%$ of children. ${ }^{30}$ There are several hypotheses about the pathophysiology of bAVM recurrence, as well as putative mechanisms to explain a higher incidence in children. Some authors have postulated a "reserve" nidus, which presumably finds expression after the primary nidus has been extirpated. ${ }^{7}$ Others have described, perhaps related, potentially unrecognized compartments at the time of treatment. ${ }^{8}$ Of course, neither of these would explain the increased rate of bAVM recurrence in children. Dysregulation of angiogenesis by vascular endothelial growth factor has been suggested as a possible cause, leading to endothelial cell activation and immature vessels sprouting from nearby normal arteries. ${ }^{9}$ Children, having higher vascular endothelial growth factor levels, stronger vascular endothelial growth factor receptor expression, and faster endothelial cell turnover, are be- lieved to be at a higher risk of this angiodysgenesis. ${ }^{10}$ Finally, the veracity of a negative findings on immediate posttreatment angiography has been questioned: Acute changes in hemodynamics, along with edema and vasospasm, could theoretically mask a residual nidus that would later be deemed recurrent. ${ }^{11}$

A recent systematic review in pediatric bAVMs showed that recurrence was detected significantly earlier in patients who underwent follow-up imaging than in those without it $(3.56 \pm 3.67$ years versus $8.86 \pm 5.61$ years; $P=.017) .{ }^{12}$ More important, there was a significant difference in the rate of presentation with rupture with follow-up versus without it $(13.3 \%$ versus $57.1 \%, P=$ $.04)$. Not surprisingly, the acceptance of MR imaging for surveillance following bAVM treatment has been widespread. ${ }^{15}$ However, there are no data in the literature regarding the predictive value of positive or negative study findings. Our results showed that while the appearance of abnormal vessels on TOFMRA had a high specificity (96\%) and PPV (86\%), it had a low sensitivity (50\%). On the other hand, the presence of abnormal enhancement on CEMRI was a poor predictor (specificity and PPV approximately $40 \%$ ) for recurrence, though the sensitivity was higher $(85 \%)$. The combined sensitivity $(75 \%)$, specificity (91\%), and PPV (86\%) values for both sequences taken together, along with noninvasiveness and absence of ionizing radiation, provide the rationale for use of MR imaging for surveillance in children after bAVM treatment and also show the complementary role of interpreting TOF-MRA and 

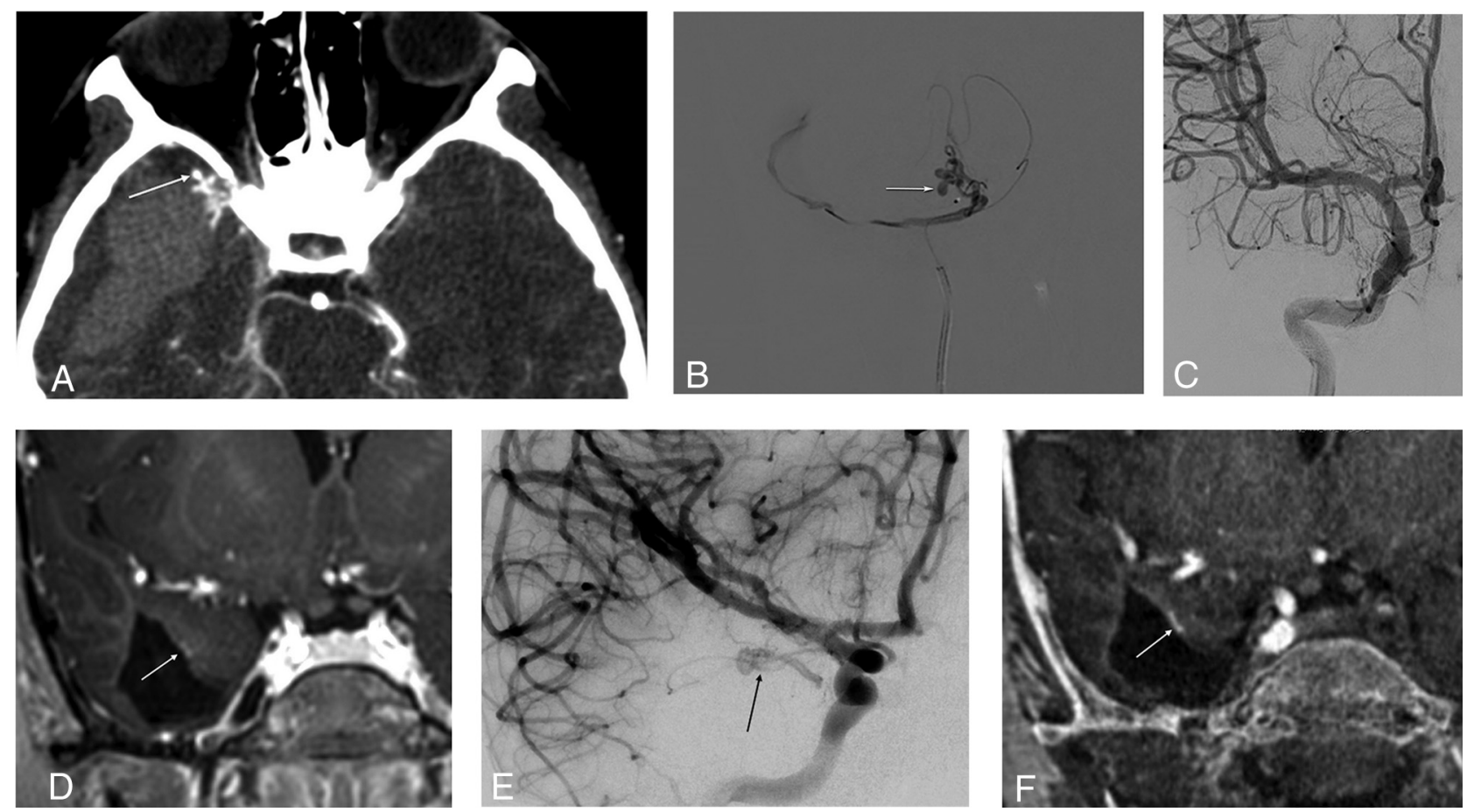

FIG 4. 3DRA-MR imaging fusion for accurate depiction of a small AVM recurrence. A, Axial CTA section in an 8-year-old child who presented with sudden reduced level of consciousness, showing a right temporal hematoma and an anterior temporal AVM with an anterolaterally directed pseudoaneurysm (arrow). B. Microcatheter injection into the temporopolar branch of the right middle cerebral artery shows the AVM and rupture point (arrow). C, This was embolized with $n$-BCA in Lipiodol, achieving a complete angiographic cure. $D$, MR imaging performed 1 year after treatment shows a small juxtamural nodular enhancement (arrow) on this postgadolinium coronal section. $E$, Basal view from a right internal carotid injection on subsequent conventional angiography confirms recurrent arteriovenous shunting (arrow). F, 3DR-MR imaging coronal fused image confirms CEMRI findings, as well as providing exquisite delineation of feeding arteries and draining vein (not shown here). This sequence, providing all requisite information for surgical resection, was used for intraoperative neuronavigation.

CEMRI findings together for these patients. However, the ability to exclude a recurrence was approximately $80 \%$ (NPV) for TOF-MRA and CEMRI, either independently or when combined.

To be useful as a screening tool for bAVM recurrence, a high NPV is critical to minimize the chance of missing patients who could have a recurrence. With $20 \%$ of recurrences not being detected on both sequences, a negative posttreatment MR imaging finding cannot completely exclude the need to perform conventional angiography. A recent study on 28 surgically resected pediatric bAVMs followed with conventional angiography showed that even an early (3-or 6-month) posttreatment conventional angiogram with negative findings could not exclude recurrence at 1 year. ${ }^{18}$ In that study, patients did not routinely undergo MR imaging surveillance, which could therefore not be compared against conventional angiography, and the authors highlighted the need for such data. Therefore, we maintain that a 1-year follow-up angiogram is imperative even in the presence of normal MR imaging examination findings, given the clinical consequences of recurrence. Although none of the recurrences in our cohort presented with hemorrhage, this outcome is likely testament to close surveillance and retreatment.

The low PPV of finding enhancement on MR imaging after bAVM treatment is not unexpected. Postsurgical nonvascular enhancement is reported after intracranial operations $s^{22,23}$ and is likely from reactive tissue or dural folds. We found that whereas a vascular "tuft" on TOF-MRA within the cavity was a strong indicator of a recurrent nidus, patchy or nodular enhancement on CEMRI was not, unless located along a resection margin away from the dural surface. Linear vascular structures on TOF-MRA coursing into the cavity were more likely to reflect recurrence, whereas linear enhancement was not a good indicator of recurrence, either within the cavity or along its margins, unless traceable into a draining vein.

MR imaging fusion of 3DRA has been previously shown to be a useful adjunct to evaluate complex neurovascular lesions in children. ${ }^{31}$ 3DRA is a volumetric angiographic study with the possibility of CT angiographic reconstruction. The ability to perform 3DRA with low radiation doses while maintaining high spatial and contrast resolution reconstructions has also been demonstrated. ${ }^{32}$ This allows the use of 3DRA-MR imaging fusion to confidently identify or exclude small vascular nidi or dysplastic structures that are characteristic of bAVM recurrence in children. This is especially relevant for false-positive MR imaging studies because MR imaging enhancement, though highly sensitive for vascular structures, is of questionable significance in the posttreatment setting, as described above. Additionally, a stack of reformatted fused images can conclusively corroborate a negative MR imaging finding. The superior 3D depiction enabled by 3DRA has been used for radiosurgical treatment planning as well as for neuronavigation of bAVMs. The ability to merge the high vascular detail 
from 3DRA and anatomic information from MR imaging makes this an ideal tool to depict small recurrences for neuronavigation. In our series, 3DRA MR imaging fusion was available and used for neuronavigation during resection of 2 bAVM nidi that recurred.

Our study had some limitations. Because the study was retrospective, patient inclusion was not consecutive and data collection was limited to available clinical and radiologic information. To minimize heterogeneity arising from reporting patterns, the study neuroradiologist analyzed all images in a blinded fashion, and another neuroradiologist analyzed 3DRA-MR imaging fusion images, both of whom have several years' experience with neurovascular imaging and angiography. In addition, several patients with treated bAVMs who had not been followed per protocol with posttreatment MR imaging and conventional angiography at our institution were excluded from the study. Therefore, our cohort, selected for the purpose of this study, did not allow reporting of true recurrence rates and resulted in a spuriously high recurrence rate $(13 / 39,33 \%)$ as a result of our study design, with individuals with positive MR imaging findings being more likely to receive close angiographic follow-up. However, it was not the objective of this study to determine recurrence rates for bAVM, which are well-documented in the literature.

We also noted a large variation in the intervals between treatment and imaging, with longer interval times before 2015 (treatment to MR imaging time: before $2015,555.3 \pm 755.8$ days; after $2015,170.3 \pm 128.9$ days; $P=.08$ ). This presumably reflects the evolution of practice patterns with time, and a progressively increasing acceptance of MR imaging as a screening tool. Another limitation was that all patients in our cohort had low grade (equal or less than grade 3) bAVMs, not allowing us to draw strong conclusions about the effect of grade on recurrence. This is as much because most pediatric bAVMs are smaller at presentation, as it is due to most larger grade bAVMs being either not amenable to therapeutic interventions or receiving staged treatment with no angiographic "cure." Finally, we did not examine data for longterm recurrence rates or cure, which, though crucial information for bAVM prognostication and follow-up, would have been limited by sample sizes being prohibitively small for this exercise. Further longitudinal studies of long-term recurrence rates are planned to determine these data.

\section{CONCLUSIONS}

TOF-MRA and CEMRI sequences provide complementary information for determining bAVM recurrence after angiographic cure in children with bAVMs and, when interpreted together, have an approximately $85 \%$ predictive value compared with conventional angiography. Certain MR imaging features can increase accuracy, but MR imaging as a surveillance tool for this population must still be supplemented by conventional angiography. Coregistering 3DRA with MR imaging increases the diagnostic confidence regarding the presence and location of bAVM recurrence and is therefore potentially suited for intraoperative neuronavigation.

\section{ACKNOWLEDGMENTS}

We would like to acknowledge all the neuroradiologists and neurosurgeons at our institution and the MR imaging, CT, and Interventional Radiology technologists who played a key role in the acquisition of images used in this study. Furthermore, we acknowledge the support of the Ontasian Imaging Lab.

Disclosures: Afsaneh Amirabadi-UNRELATED: Employment: The Hospital for Sick Children. Manohar M. Shroff-UNRELATED: Expert Testimony: Canadian Medical Protective Association or plaintiff lawyer, Comments: occasional expert testimony in abusive head trauma or neonatal hypoxic ischemic encephalopathy, none so far in the matter of brain AVM and its imaging. Timo Krings_UNRELATED: Consultancy: Stryker, Medtronic; Royalties: Thieme, Comments: principal author of 2 books on neuroradiology.

\section{REFERENCES}

1. Stapf C, Mast H, Sciacca RR, et al. Predictors of hemorrhage in patients with untreated brain arteriovenous malformation. Neurology 2006;66:1350-55 CrossRef Medline

2. Rutledge WC, Ko NU, Lawton MT, et al. Hemorrhage rates and risk factors in the natural history course of brain arteriovenous malformations. Transl Stroke Res 2014;5:538-42 CrossRef Medline

3. van Beijnum J, van der Worp HB, Buis DR, et al. Treatment of brain arteriovenous malformations: a systematic review and meta-analysis. JAMA 2011;306:2011-19 CrossRef Medline

4. Blauwblomme T, Bourgeois M, Meyer P, et al. Long-term outcome of 106 consecutive pediatric ruptured brain arteriovenous malformations after combined treatment. Stroke 2014;45:1664-71 CrossRef Medline

5. Beslow LA, Licht DJ, Smith SE, et al. Predictors of outcome in childhood intracerebral hemorrhage: a prospective consecutive cohort study. Stroke 2010;41:313-18 CrossRef Medline

6. Gross BA, Du R. Natural history of cerebral arteriovenous malformations: a meta-analysis. J Neurosurg 2013;118:437-43 CrossRef Medline

7. Caplan JM, Yang W, Garzon-Muvdi T, et al. 120 rates of re-hemorrhage, risk factors, and outcomes of previously ruptured arteriovenous malformations (AVMs). Neurosurgery 2017;64(CN_ suppl_1):226-26 CrossRef

8. Mohr JP, Parides MK, Stapf C, et al; international ARUBA investigators. Medical management with or without interventional therapy for unruptured brain arteriovenous malformations (ARUBA): a multicentre, non-blinded, randomised trial. Lancet 2014;383:614-21 CrossRef Medline

9. Elhammady MS, Heros RC. Editorial: the ARUBA study-where do we go from here? J Neurosurg 2017;126:481-85 CrossRef Medline

10. Wong J, Slomovic A, Ibrahim G, et al. Microsurgery for ARUBA trial (A Randomized Trial of Unruptured Brain Arteriovenous Malformation)-eligible unruptured brain arteriovenous malformations. Stroke 2017;48:136-44 CrossRef Medline

11. Mohr JP, Hartmann A, Kim H, et al. Viewpoints on the ARUBA trial. AJNR Am J Neuroradiol 2015;36:615-17 CrossRef Medline

12. Morgenstern PF, Hoffman CE, Kocharian G, et al. Postoperative imaging for detection of recurrent arteriovenous malformations in children. J Neurosurg Pediatr 2016;17:134-40 CrossRef Medline

13. Javadpour M, Al-Mahfoudh R, Mitchell PS, et al. Outcome of microsurgical excision of unruptured brain arteriovenous malformations in ARUBA-eligible patients. Br J Neurosurg 2016;30:619-22 CrossRef Medline

14. Joyce C, Gomez CR. Reimagining ARUBA: theoretical optimization of the treatment of unruptured brain arteriovenous malformations. J Stroke Cerebrovasc Dis 2018;27:3100-07 CrossRef Medline

15. Jimenez JE, Gersey ZC, Wagner J, et al. Role of follow-up imaging after resection of brain arteriovenous malformations in pediatric patients: a systematic review of the literature. J Neurosurg Pediatr 2017;19:149-56 CrossRef Medline 
16. Geibprasert S, Pongpech S, Jiarakongmun P, et al. Radiologic assessment of brain arteriovenous malformations: what clinicians need to know. Radiographics 2010;30:483-501 CrossRef Medline

17. Tranvinh E, Heit JJ, Hacein-Bey L, et al. Contemporary imaging of cerebral arteriovenous malformations. AJR Am J Roentgenol 2017; 208:1320-30 CrossRef Medline

18. Lang SS, Beslow LA, Bailey RL, et al. Follow-up imaging to detect recurrence of surgically treated pediatric arteriovenous malformations. J Neurosurg Pediatr 2012;9:497-504 CrossRef Medline

19. Gross BA, Frerichs KU, Du R. Sensitivity of CT angiography, T2weighted MRI, and magnetic resonance angiography in detecting cerebral arteriovenous malformations and associated aneurysms. J Clin Neurosci 2012;19:1093-35 CrossRef Medline

20. Chowdhury AH, Ghose SK, Mohammad QD, et al. Digital subtraction angiography is superior to magnetic resonance angiography in diagnosis of cerebral arteriovenous malformation. Mymensingh Med J 2015;24:356-65 Medline

21. Lee CC, Reardon MA, Ball BZ, et al. The predictive value of magnetic resonance imaging in evaluating intracranial arteriovenous malformation obliteration after stereotactic radiosurgery. J Neurosurg 2015;123:136-44 CrossRef Medline

22. Lescher S, Schniewindt S, Jurcoane A, et al. Time window for postoperative reactive enhancement after resection of brain tumors: less than 72 hours. Neurosurg Focus 2014;37:E3 CrossRef Medline

23. Bette S, Gempt J, Huber T, et al. Patterns and time dependence of unspecific enhancement in postoperative magnetic resonance imaging after glioblastoma resection. World Neurosurg 2016;90: 440-47 CrossRef Medline

24. Gulani V, Calamante F, Shellock FG, et al. Gadolinium deposition in the brain: summary of evidence and recommendations. Lancet $\mathrm{Neu}$ rol 2017;16:564-70 CrossRef Medline

25. Semelka RC, Ramalho J, Vakharia A, et al. Gadolinium deposition disease: Initial description of a disease that has been around for a while. Magn Reson Imaging 2016;34:1383-90 CrossRef Medline

26. Guo BJ, Yang ZL, Zhang LJ. Gadolinium deposition in brain: current scientific evidence and future perspectives. Front Mol Neurosci 2018;11:335 CrossRef Medline

27. Terashima KH, Reich DS. Gadolinium deposition: practical guidelines in the face of uncertainty. Lancet Neurol 2017;16:495-97 CrossRef Medline

28. Aboukaïs R, Vinchon M, Quidet M, et al. Reappearance of arteriovenous malformations after complete resection of ruptured arteriovenous malformations: true recurrence or false-negative early postoperative imaging result? J Neurosurg 2017;126:1088-93 CrossRef Medline

29. Freudenstein D, Duffner F, Ernemann U, et al. Recurrence of a cerebral arteriovenous malformation after surgical excision. Cerebrovasc Dis 2001;11:59-64 CrossRef Medline

30. Sorenson TJ, Brinjikji W, Bortolotti C, et al. Recurrent brain arteriovenous malformations (AVMs): a systematic review. World Neurosurg 2018;116:e856-66 CrossRef Medline

31. Muthusami P, Shkumat N, Rea V, et al. CT reconstruction and MRI fusion of 3D rotational angiography in the evaluation of pediatric cerebrovascular lesions. Neuroradiology 2017;59:625-33 CrossRef Medline

32. Shkumat NA, Shroff MM, Muthusami P. Radiation dosimetry of 3D rotational neuroangiography and 2D-DSA in children. AJNR Am J Neuroradiol 2018;39:727-33 CrossRef Medline 